\title{
Temperature-Dependent Emission Kinetics of Colloidal Semiconductor Nanoplatelets Strongly Modified by Stacking
}

\author{
Onur Erdem, ${ }^{\dagger}$ Murat Olutas, $^{\dagger, \dagger}$ Burak Guzelturk, $^{\dagger}$ Yusuf Kelestemur, $^{\dagger}$ and Hilmi Volkan Demir ${ }^{*}, \dagger, \S$ \\ ${ }^{\dagger}$ Department of Electrical and Electronics Engineering, Department of Physics, UNAM - Institute of Materials Science and \\ Nanotechnology, Bilkent University, Ankara 06800, Turkey \\ ${ }^{*}$ Department of Physics, Abant Izzet Baysal University, Bolu 14280, Turkey \\ ${ }^{\S}$ LUMINOUS! Center of Excellence for Semiconductor Lighting and Displays, School of Electrical and Electronic Engineering, \\ School of Physical and Mathematical Sciences, Nanyang Technological University, Nanyang Avenue, Singapore 639798, Singapore
}

Supporting Information

\begin{abstract}
We systematically studied temperature-dependent emission kinetics in solid films of solution-processed CdSe nanoplatelets (NPLs) that are either intentionally stacked or nonstacked. We observed that the steady-state photoluminescence (PL) intensity of nonstacked NPLs considerably increases with decreasing temperature, whereas there is only a slight increase in stacked NPLs. Furthermore, PL decay time of the stacked NPL ensemble is comparatively much shorter than that of the nonstacked NPLs, and this result is consistent at all temperatures. To account for these observations, we developed a probabilistic model that describes excitonic processes in a stack using Markov chains, and we found excellent agreement between the model and experimental results. These findings develop the insight that the competition between the radiative channels and energy transferassisted hole trapping leads to weakly temperature-dependent PL intensity in the case of the stacked NPL ensembles as compared to the nonstacked NPLs lacking strong energy transfer. This study shows that it is essential to account for the effect of NPL stacking to understand their resulting PL emission properties.
\end{abstract}

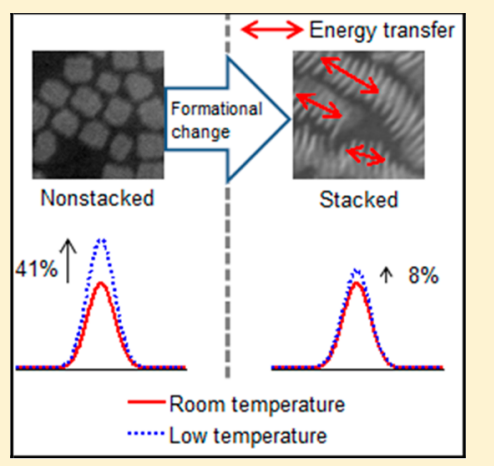

S emiconductor nanoplatelets (NPLs), also known as colloidal quantum wells (QWs), have been most recently introduced as a new class of solution-processed nanocrystals. These NPLs are atomically flat nanostructures with a magic sized vertical thickness and relatively large lateral dimensions, which generally vary in the range of $10-100 \mathrm{~nm} .{ }^{1-3}$ Because the lateral dimensions are typically larger than the exciton Bohr radii, ${ }^{4}$ the quantum confinement in the NPLs is tight in only one dimension. To date, NPLs have been shown to exhibit giant oscillator strength transition (GOST) ${ }^{1}$ accompanied by very narrow photoluminescence $(\mathrm{PL})$ emission line width as narrow as $8 \mathrm{~nm}(<40 \mathrm{meV}){ }^{5,6}$ These favorable properties make NPLs highly attractive for device applications including lightemitting diodes ${ }^{7}$ and lasers. ${ }^{8-10}$

One important issue related to the NPLs, however, is their self-assembly in solid films, commonly regarded as stacking, that is believed to result from van der Waals forces building because of large and flat surface area of the NPLs, favoring parallel alignment of the flat faces in the long needlelike NPL chains. ${ }^{11}$ The phenomenon of stacking in NPLs has been observed and reported by several different studies. ${ }^{2,6,11,12}$ It has been demonstrated that the optical properties of the stacked NPL ensembles differ from those of nonstacked ones. For instance, NPL stacks were shown to emit polarized light. ${ }^{11}$ Also, ultrafast exciton transfer in the stacked NPL assemblies enabled by efficient Förster resonance energy transfer (FRET) was uncovered in a previous study of ours. ${ }^{6}$ The exciton transport along the NPL stacks was found to lead to strong PL quenching through charge trapping in the defected NPL subpopulations. ${ }^{6}$ In another study, stacked NPLs have been reported to exhibit a newly emerging PL peak at the lowerenergy tail of the emission at cryogenic temperatures, which was attributed to the phonon-line replica. ${ }^{2}$ However, temperature-dependence of transient and steady-state PL emission properties of the stacked NPL ensembles have not yet been systematically studied or understood; neither has the effect of stacking on the temperature-dependent PL emission kinetics of NPLs been elucidated to date, although stacking alters the excitonic processes in NPLs in a substantial way.

In this work, we systematically investigated and comparatively studied time-resolved and steady-state emission of stacked versus nonstacked NPL ensembles as a function of the temperature. We reveal that the temperature-dependent evolution of steady-state PL intensity and transient PL decay fundamentally differs between nonstacked and stacked NPLs. We show that these differences can be accounted for by exciton transfer-induced charge trapping in the stacked NPLs. We model the exciton transfer among the NPLs within a stack as a Markovian process to estimate the quantum yield (QY) and PL lifetime in stacked NPL ensembles, which shows excellent

Received: December 13, 2015

Accepted: January 20, 2016

Published: January 20, 2016 


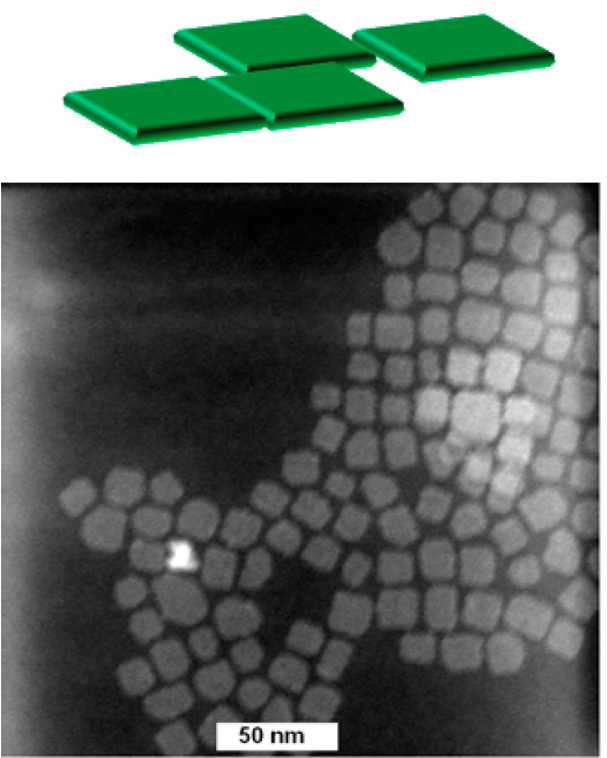

(a)

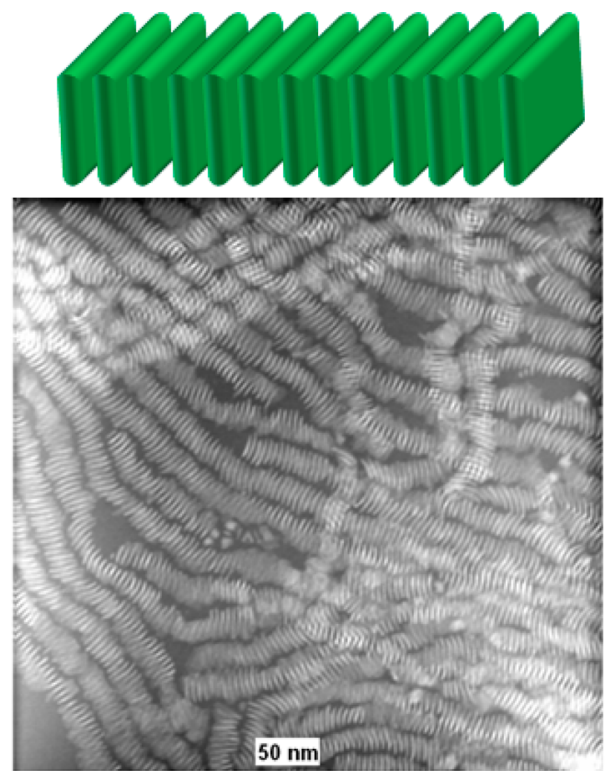

(b)

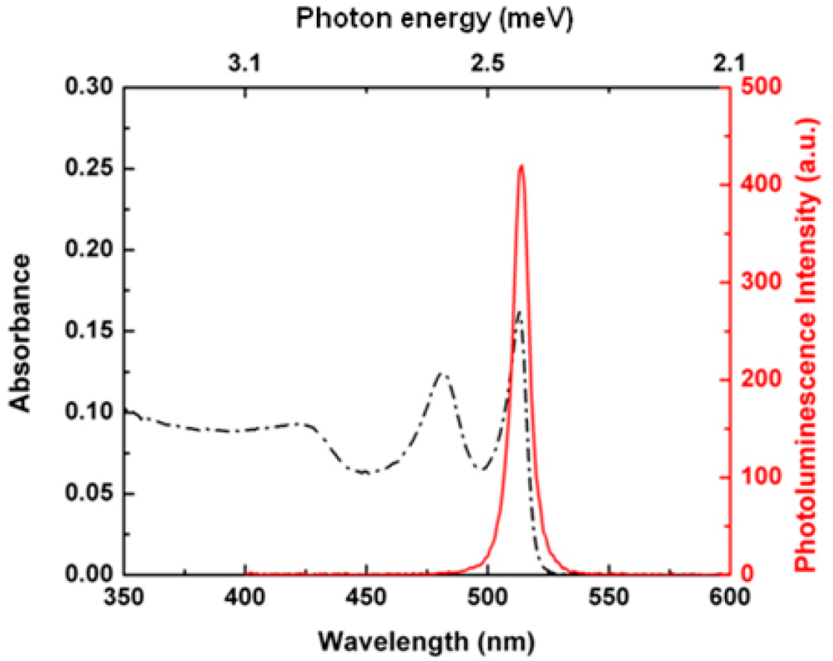

(c)

Figure 1. HAADF-STEM images of (a) nonstacked and (b) stacked 4 ML NPLs and their schematic representation. (c) Room-temperature absorption (dot-dashed line) and emission spectra (solid line) of the nonstacked 4 ML CdSe NPLs dissolved in hexane.

agreement with the experimental results. These findings uncover the strong competition between exciton trapping and radiative recombination processes as a function of the temperature leading to the observed differences in PL dynamics of the stacked and nonstacked NPL ensembles. This understanding will be crucial for tailoring NPL ensembles in highly efficient light-emitting diodes and lasers.

In this study, we synthesized and used CdSe NPLs with a vertical thickness of 4 monolayers (MLs) of lattice unit. The detailed synthesis procedure of the NPLs is explained in the Experimental Section. To induce stacking in the NPL ensemble, a cleaning procedure with ethanol was applied after the synthesis. The different configurations of NPLs in two different ensembles were verified via high-angle annular darkfield scanning transmission electron microscopy (HAADFSTEM) images shown in panels a and b of Figure 1, which display the CdSe NPLs in the nonstacked and stacked formation, respectively. As seen in Figure 1a, the nonstacked NPLs are flat-lying on the TEM grid and are well-separated, and there is no indication of stacking. However, the stacked NPLs are aligned face-to-face and standing on their edges instead of lying flat as seen in Figure $1 \mathrm{~b}$. It was observed that the length of a NPL stack can be as long as $1 \mu \mathrm{m}$, which corresponds to about 230 NPLs in a stack. Longer stacks are also possible (see Figure S2 for a histogram of the number of NPLs in stacks). The absorbance and PL spectra of 4 ML CdSe NPLs in solution are shown in Figure 1c. Two peaks observed at 480 and $512 \mathrm{~nm}$ in the absorption spectrum correspond to the electron-light hole and electron-heavy hole transitions, respectively. The PL emission peak is at $514 \mathrm{~nm}$, and the full width at half-maximum (fwhm) of the PL emission spectrum is $9 \mathrm{~nm}(\sim 42 \mathrm{meV})$ at room temperature (RT).

To carry out the temperature-dependent time-resolved fluorescence (TRF) and steady-state PL measurements, we prepared solid films of NPL ensembles on quartz substrates, which are $1.2 \times 1.2 \mathrm{~cm}^{2}$ in size. We used a time-correlated single-photon-counting (TCSPC) system (PicoHarp 300) to make transient fluorescence measurements and a spectrometer 

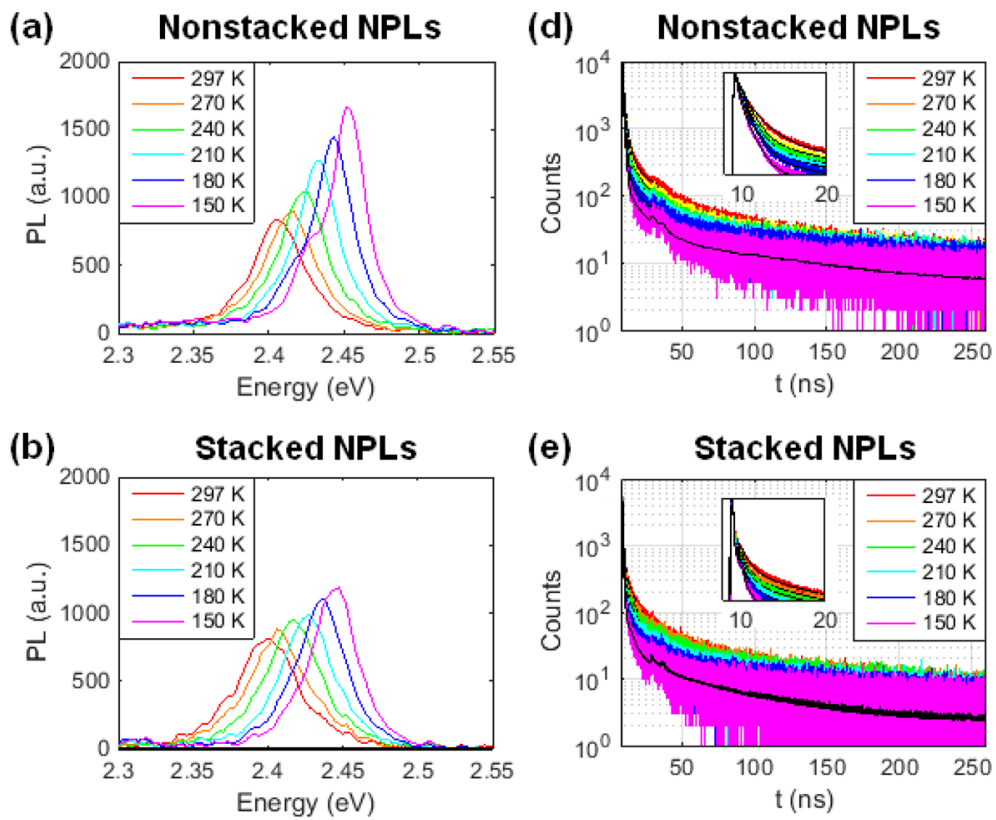

(c)

(f)
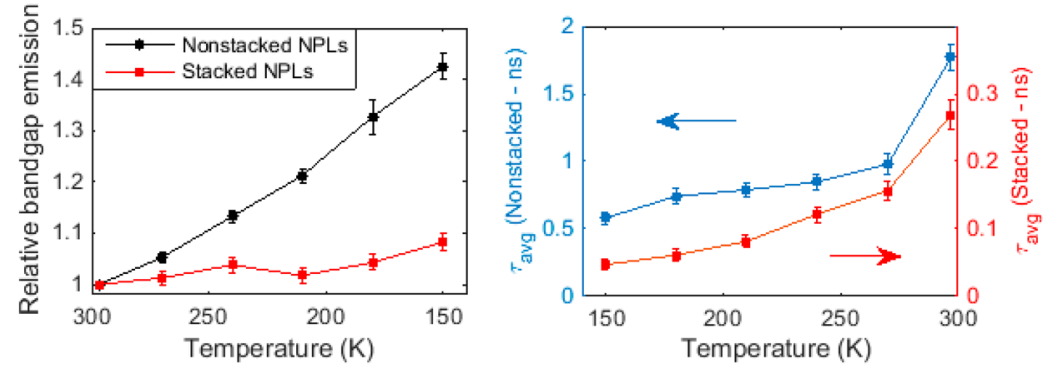

Figure 2. Photoluminescence (PL) emission spectra of the (a) nonstacked NPLs and (b) stacked NPLs at different temperatures. The slight distortion in the PL signal is due to noise in the spectrometer. (c) Change in PL intensity relative to that at room temperature for the nonstacked (black circles) and stacked (red triangles) NPL ensembles. Time-resolved photoluminescence decays of the (d) nonstacked and (e) stacked NPL ensembles. Black lines show the fits to the decay curves. Insets show the same decay curves in a shorter time window. Scales of the $y$ axes of the inset are from 100 to 10000 . (f) Amplitude-averaged lifetimes $\left(\tau_{\text {avg }}\right)$ of the nonstacked (blue squares) and stacked (red squares) ensembles.

to record the steady-state PL spectrum. The temperaturedependent TRF measurements were performed using a closedcycle $\mathrm{He}$ cryostat, at temperatures ranging from room temperature to $150 \mathrm{~K}$. Figure 2 depicts the results of temperature-dependent PL measurements of both the nonstacked and stacked NPL ensembles. The steady-state PL spectra at different temperatures for the nonstacked and stacked ensembles are shown in panels a and b of Figure 2, respectively.

The PL emission peak of the nonstacked NPL ensemble at $\mathrm{RT}$ is $2.41 \mathrm{eV}(\sim 514 \mathrm{~nm})$ and it blue-shifts by about $8 \mathrm{~nm}$ to $2.45 \mathrm{eV}(\sim 506 \mathrm{~nm})$ at $150 \mathrm{~K}$. In addition, the fwhm of the PL emission narrows to $25 \mathrm{meV}(5.1 \mathrm{~nm})$ at $150 \mathrm{~K}$, whereas it is $41 \mathrm{meV}(8.8 \mathrm{~nm})$ at RT. In the case of stacked NPLs, the PL emission peak shifts from 2.40 to $2.44 \mathrm{eV}$ while the fwhm of the $\mathrm{PL}$ emission decreases from 47.3 to $35.1 \mathrm{meV}$ as the temperature is decreased from RT to $150 \mathrm{~K}$. The slight red shift of the PL peak and wider fwhm in the stacked NPL ensemble might be caused by different effective dielectric medium seen by the NPLs due to the dense packing of NPLs in stacks. On the other hand, at temperatures below $180 \mathrm{~K}$, we observe an additional PL emission feature, which is red-shifted as compared to the main emission peak (see Figure S1). The difference between the maxima of these two features is $26 \mathrm{meV}$. The contribution of this second peak to the bandgap emission increases further at lower temperatures (see Figure S1). A similar feature in the PL emission spectrum of NPLs has been reported by Tessier et al. for the stacked NPLs, with the additional peak appearing only at cryogenic temperatures and $25 \mathrm{meV}$ difference between the two peaks. ${ }^{2}$ The occurrence of this second peak was attributed to the phonon-line emission. In this study, however, we have observed this additional spectral feature only for the nonstacked ensemble and not for the stacked one. The investigation of the emergence of this second peak is in progress and beyond the scope of this current work.

Figure $2 \mathrm{c}$ shows the change in the PL emission intensity for the nonstacked and stacked NPLs as a function of the temperature. For the nonstacked NPL ensemble, the bandgap emission increases by more than $40 \%$ when the temperature is reduced from 300 to $150 \mathrm{~K}$, whereas the increase is only $8 \%$ in the stacked ensemble over the same temperature range. The increase in PL emission of the nonstacked ensemble when the temperature is reduced is in agreement with the literature, and it has been attributed to GOST. ${ }^{1}$ In addition, panels $\mathrm{d}$ and e of Figure 2 depict the transient PL decays for the nonstacked and stacked NPL ensembles, respectively. The feature at about 30 ns in the decay curves is due to the afterpulsing in the detector and is accounted for by fitting the decays in the reconvolution mode. The decays were numerically fitted with four exponential decay functions due to their complex decay kinetics. The 

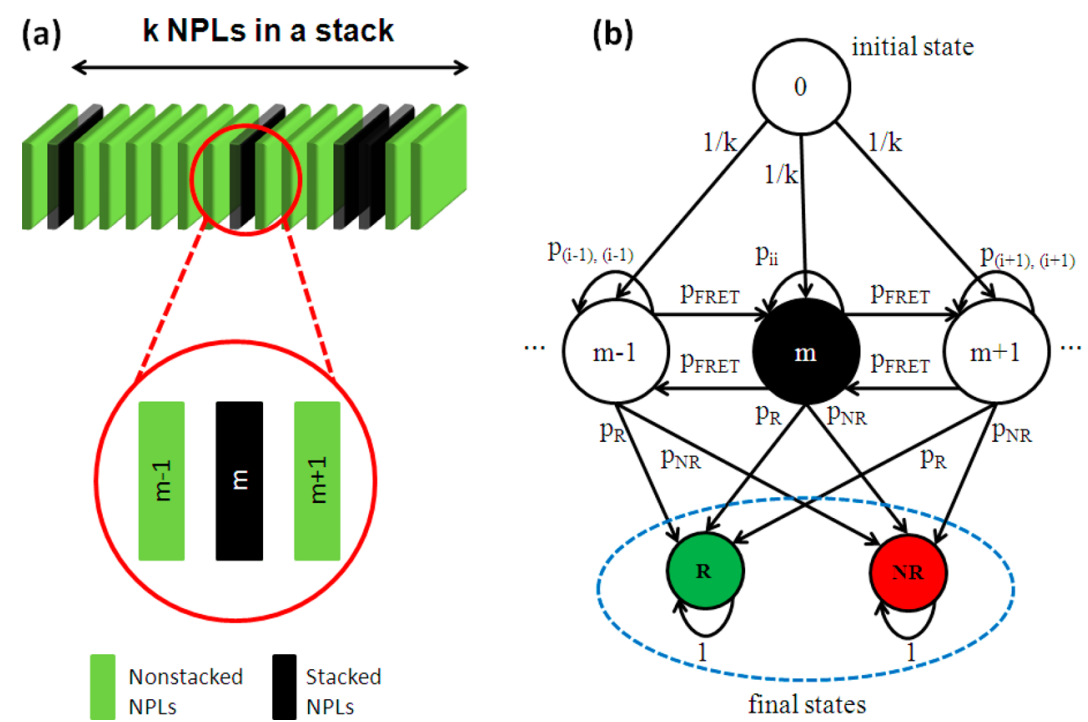

Figure 3. (a) Illustration of an exemplary NPL stack with some of its NPLs defected (shown in black) while the rest are nondefected (shown in green). (b) Part of the Markov chain used to model excitonic transition probabilities in the NPL stack drawn in and a. States 1 to $k$ represent an exciton being in a corresponding NPL in the stack (only $m-1, m$, and $m+1$ are shown here). $k$ is the number of NPLs in the stack. Black circles represent states corresponding to defected NPLs. The system will eventually end up in either R (radiative) or NR (nonradiative) state, corresponding to radiative and nonradiative recombination, respectively. Transition probabilities are determined by the transition rates and the time step, $\Delta t$.

multiexponential decay behavior for either a single NPL or an ensemble of NPLs was previously reported. ${ }^{1,5,10}$ Owing to their complex decay dynamics, here we used amplitude-averaged lifetimes to analyze the transient PL kinetics of the NPL ensembles, which is appropriate in the energy-transfer studies when the emission of the donor alone or the emission of the donor in the presence of acceptor has a complex decay. ${ }^{13}$ Figure $2 \mathrm{f}$ exhibits the amplitude-averaged PL lifetimes $\left(\tau_{\text {avg }}\right)$ of the decays for the nonstacked and stacked NPL ensembles as a function of temperature. The $\tau_{\text {avg }}$ of the nonstacked NPL ensemble was found to be $1.8 \mathrm{~ns}$ at RT and $0.58 \mathrm{~ns}$ at $150 \mathrm{~K}$, whereas it is only $270 \mathrm{ps}$ at RT and reduces to $47 \mathrm{ps}$ at $150 \mathrm{~K}$ for the stacked NPL ensemble. The TRF measurements have been conducted by collecting the emission at the peak wavelength at each temperature. However, the decays at red and blue tails of the bandgap emission feature also have similar average lifetimes (see Figure S3), suggesting the absence of inhomogeneous broadening. The decays get progressively faster with decreasing temperature, and it becomes unfavorable to perform a thorough and reliable lifetime analysis for the stacked NPLs below $150 \mathrm{~K}$ because the fwhm of the decay becomes comparable to that of the instrument response.

Here, we have observed that the increase in PL intensity of stacked NPL ensembles at lower temperatures is suppressed to a great extent, in contrast to the increase of PL intensity of nonstacked NPLs at lower temperatures. To explain the differences between the temperature-dependent PL evolution of the stacked and nonstacked NPL ensembles, we consider homo-FRET ${ }^{14}$ along with occasional charge trapping in the NPLs. As we have previously revealed, homo-FRET can take place at a very high rate between the neighboring NPLs in stacks. ${ }^{6,15}$ It has also been reported by previous studies on NPLs that a certain fraction of a NPL population contains hole traps that causes exciton quenching., ${ }^{3,16}$ As a result, some of the excitons in a NPL chain, which are initially in a NPL with no trapping sites (nondefected NPLs), may transfer their energy via FRET to a NPL having a trap site (defected NPL), in which the likelihood of exciton quenching is much higher. In general, mathematical models for the kinetics of excitation transport in excitation lattices have been studied for various applications, including excited molecule dynamics in the photosynthetic units, dye solutions composed of monomers and dimers, and closely packed quantum dot ensembles. ${ }^{17-19}$ In these systems, some of the excitation sites in the lattice that can quench the excitation alter the excitation dynamics. Apart from the aforementioned studies, purely theoretical models have also been previously developed to understand excitation kinetics in a lattice having traps. ${ }^{20-22}$ Here, we developed a mathematical model, which regards the exciton transport within the NPL stack as a random walk and uses Markov chains to estimate the changes in the PL lifetime and QY of the NPLs in stacked configuration. Figure 3 schematically illustrates how we model the excitonic transitions in a NPL stack as a Markov chain. For each NPL ensemble, such as the one illustrated in Figure 3a, we define a Markov chain as in Figure 3b. We use the states of the Markov chain to define the position of an exciton as well as the associated recombination events. Given the QY of NPLs in nonstacked form; the locations of defected NPLs; and rates for radiative and nonradiative recombination, charge trapping, and FRET, we can estimate the probability and duration of survival of an exciton in the stack using the transition probability matrices, $\mathbf{P}_{i}$, for stacks in the ensemble. We use the temperature-dependent experimental data on the nonstacked NPLs as an input to our model to semiempirically determine the temperature-dependent $\mathrm{QY}$ and rates for exciton recombination and FRET. Locations of the defected NPLs are determined randomly in the simulations, and the trapping rate is used as a variational parameter.

Once the transition probability matrices are determined, the calculations yield (see Supporting Information for the detailed derivation) that the QY of the stacked NPL ensemble can be expressed as 

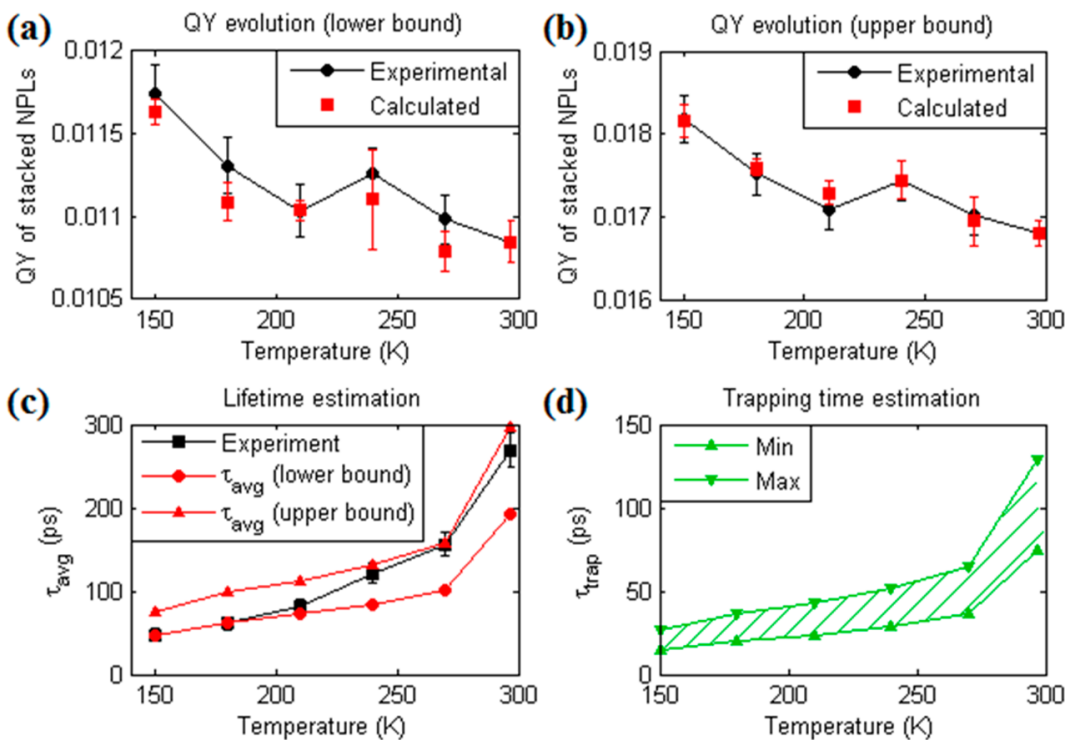

Figure 4. Estimated lifetimes and quantum yields of stacked NPL ensembles for a defected PL subpopulation fraction of $f=0.4$ and $\mathrm{QY} \mathrm{RT}_{\mathrm{RT}}=10 \%$. Estimation of the QY evolution with temperature for (a) lower bound and (b) upper bound of the QY. (c) Estimation of PL lifetimes for the assumed values of room-temperature $\mathrm{QY}$ of the stacked NPLs in panels a and $\mathrm{b}$. The experimental data (black squares) is also given together with the upper (red triangles) and lower bound (red squares) estimations. (d) Estimations for the trapping time for the assumed values of the roomtemperature QY of stacked NPLs in panels a and $\mathrm{b}$. The trapping time versus temperature curve is estimated to lie within the shaded area in panel $\mathrm{d}$.

$$
\mathrm{QY}=\frac{\sum_{i} k_{i} \lim _{n \rightarrow \infty}\left(P_{i}^{n}\right)_{0, R}}{\left(\sum_{i} k_{i}\right)}
$$

where $\left(P_{i}^{n}\right)_{0, R}$ is the probability of radiative recombination in the ith NPL stack in $n$ time steps. The amplitude-averaged PL lifetime of the stacked NPL ensembles can be estimated by

$$
\tau_{\text {avg }}=\frac{\sum_{i} k_{i} \lim _{n \rightarrow \infty}\left(P_{i}^{n}\right)_{0, R}}{\left(\sum_{i} k_{i}\right)\left(\sum_{i}\left(P_{i}^{2}\right)_{0, R}\right)} \Delta t
$$

where $\Delta t$ is the small time step between two discrete time instants.

Our mathematical model assumes that there is at most one exciton in a NPL stack at any time instant. To ensure the validity of this assumption, we used a sufficiently low laser intensity with a large incidence spot with an area of $\sim 3.5 \times$ $10^{-3} \mathrm{~cm}^{2}$. Our calculations yield that the excitation laser has about $2.91 \times 10^{7}$ photons per pulse. Using $\langle N\rangle=f_{\mathrm{p}} \times \sigma$, where $f_{\mathrm{p}}$ is the per-pulse fluence and $\sigma=3.1 \times 10^{-14} \mathrm{~cm}^{2}$ is the absorption cross section of the 4 ML CdSe NPLs at $400 \mathrm{~nm},{ }^{23}$ we calculate the average exciton density per NPL as $2.90 \times$ $10^{-4}$. Even for the longest stacks observed in the TEM images (stacks with $~ 280$ NPLs, see Figure S2), the average number of excitons in a stack is smaller than 0.08 , which justifies our assumption. Moreover, the repetition period of the laser is at least $12.5 \mathrm{~ns}$, which is a sufficiently large duration for an exciton in a NPL stack to decay, so consecutive laser pulses cannot create multiple excitons in a stack.

There are two important parameters in our model that govern the dynamics; these are the room-temperature $\mathrm{QY}$ $\left(\mathrm{QY}_{\mathrm{RT}}\right)$ of nonstacked NPLs and the fraction of the defected NPLs in the NPL ensemble $(f)$. We test our model by carrying out a parametric study for possible values of $\mathrm{QY}_{\mathrm{RT}}$ for nonstacked NPLs as well as different $f$ values. The tested $\mathrm{QY}_{\mathrm{RT}}$ values for the nonstacked NPLs range from $2 \%$ to its maximum possible value, $16 \%$ (see Supporting Information). $f$ has been swept between $10 \%$ and $90 \%$. We employ Förster's theory to estimate the rate of energy transfer between neighboring NPLs in a stack. ${ }^{24}$ The calculated FRET rates vary between (23.8 $\mathrm{ps})^{-1}$ and $(3.0 \mathrm{ps})^{-1}$, which are in agreement with recently reported FRET times of 6-23 $\mathrm{ps}^{25}$ Therefore, for a total of 8 possible $\mathrm{QY}_{\mathrm{RT}}$ values and 9 possible $f$ values, i.e., a total of 72 cases tested, we carried out the computational simulations for the stacked ensemble PL lifetime and QY. As an example, the results for one of those cases, in which $\mathrm{QY}$ of the nonstacked NPLs at RT is taken as 0.1 and $f$ as 0.4, are shown in Figure 4. In this case, the $\mathrm{QY}_{\mathrm{RT}}$ for the stacked NPLs has been estimated to be between $1.09 \%$ and $1.68 \%$. The calculated $\mathrm{QY}$ at lower temperatures based on the estimation that the $\mathrm{QY}_{\mathrm{RT}}=1.09$ is presented in Figure 4a and that for $\mathrm{QY}_{\mathrm{RT}}=1.68 \%$ is given in Figure $4 \mathrm{~b}$. The corresponding PL lifetime calculations, based on the stacked $\mathrm{QY}_{\mathrm{RT}}$ estimations, are plotted in Figure 4c, together with the experimentally determined PL lifetimes. As can be seen in the figure, the experimental PL lifetimes stay inside the region bounded by the upper and lower bound estimations for the temperature-dependent PL lifetime. Finally, in Figure 4d, we show the trapping times, $\tau_{\text {trap }}$, that are used in our mathematical model to calculate the PL QY and emission lifetimes. $\tau_{\text {trap }}$ is used as an adjustable parameter that is allowed to vary with temperature. When $f=0.2-0.3$, the roomtemperature values of $\tau_{\text {trap }}$ are in agreement with the reported values of trapping time for CdSe NPLs ${ }^{16,26}$ (see Figure S4), which suggests that the actual fraction of defected NPLs in an ensemble is close to these percentages.

The results summarized in Figure 4 can be obtained and plotted for different starting combinations of the parameters $f$ and nonstacked $\mathrm{QY}_{\mathrm{RT}}$. Except the cases with $f=0.1$, for which the calculations did not converge to the experimental data, we are able to estimate the upper and lower bounds for the temperature-dependent PL lifetime and show that the experimentally determined PL lifetimes indeed stay inside the range determined by the bounds. This mathematical model thus provides a tool to calculate the PL lifetimes at a given 
temperature, as well as to estimate the temperature-dependent exciton trapping rates in the defected NPLs.

One important observation about the temperature dependence of the trapping time, $\tau_{\text {trap }}$ is that it is monotonically reduced with the decreasing temperature. This can be explained by the reduction of the thermal energy of charge carriers at low temperatures. ${ }^{27}$ With smaller thermal energy, excitons become more likely to get trapped in the defected NPLs. Over 5-fold increase from RT to $20 \mathrm{~K}$ across the wide emission feature coming from the trap sites also suggests faster charge trapping at lower temperatures (see Figure S1 and Table S1). Moreover, the increasing GOST might cause faster charge trapping at lower temperatures. It has been verified by previous studies on core/crown NPLs that the passivation of the peripheral surfaces with the growth of a crown along the lateral directions increases the QY to a great extent. ${ }^{28-30}$ This indicates that the surface traps mostly originate from the peripheral surfaces rather than wide flat surfaces. As a result, these trapping sites are more likely to trap charges when the excitonic motion is faster and the wave function spreads across the NPL. Because the giant oscillator strength is proportional to the area covered by the center of mass motion of the exciton, ${ }^{31}$ excitons should be more easily trapped when their GOST is larger at lower temperatures.

It should also be noted that the radiative recombination rate, which increases at lower temperatures as verified by the TRF measurements, are in competition with the charge trapping assisted by FRET along the NPL stacks. In the nonstacked ensemble, FRET is negligible and the radiative recombination becomes more and more dominant compared to nonradiative processes, resulting in $\mathrm{PL}$ enhancement at lower temperatures. In the stacked ensembles, however, the charge trapping is strongly assisted by FRET; therefore, there is a strong competition between the radiative recombination and charge trapping at each temperature, which explains why the temperature-dependent trends in the steady-state PL emission and the transient PL lifetimes differ for the nonstacked and stacked NPLs. The charge-trapping time, QY, and PL lifetimes of the NPLs are different at each temperature. Even though the QY of the nonstacked NPLs monotonically increase with decreasing temperature, the FRET-assisted charge trapping tends to suppress the increase in the QY, which results in the observed nonmonotonic dependence on temperature of the QY in stacked NPLs.

In summary, we systematically investigated the temperaturedependent time-resolved emission kinetics of stacked NPL ensembles at temperatures ranging from RT to $150 \mathrm{~K}$ and compared them with the nonstacked NPL ensembles. The PL decay of the stacked NPLs is observed to be much faster than those of the nonstacked NPLs at all temperatures. Moreover, the nonstacked NPLs display a clear enhancement of more than $40 \%$ in their PL emission at $150 \mathrm{~K}$, while the increase in the PL emission for the stacked NPL ensembles is only about $8 \%$. To explain the stark difference between the changes in PL lifetime and intensity of the stacked and nonstacked NPL ensembles with respect to temperature, we developed a model that takes fast FRET between neighboring NPLs and exciton trapping in the defected NPLs into account. We used this model to estimate the evolution of PL lifetime and steady-state PL intensity of NPL stacks over temperature, which show excellent agreement with the experimental data, and predicted the charge-trapping rates. These findings shed light on the temperature-dependent excitonics of the stacked NPLs and explain major modifications observed in the emission kinetics resulting from stacking.

\section{EXPERIMENTAL SECTION}

Synthesis of $4 \mathrm{ML}$ CdSe NPLs. Cadmium myristate $\mathrm{Cd}(\mathrm{Mry})_{2}$ (340 mg), selenium (Se) powder $(24 \mathrm{mg})$, and octadecene (ODE) $(30 \mathrm{~mL})$ were loaded into a $100 \mathrm{~mL}$ three-neck flask. The mixed solution was degassed under vacuum at RT for $1 \mathrm{~h}$, and it was heated to $240{ }^{\circ} \mathrm{C}$ under argon (Ar) atmosphere. Cadmium acetate dihaydrate $\mathrm{Cd}(\mathrm{OAc})_{2}(20 \mathrm{mg})$ was introduced swiftly into the reaction at $195{ }^{\circ} \mathrm{C}$. After $10 \mathrm{~min}$ of growth of CdSe NPLs at $240{ }^{\circ} \mathrm{C}$, the reaction was stopped and cooled to RT with the injection of $1 \mathrm{~mL}$ of oleic acid (OA). The resulting $4 \mathrm{ML}$ CdSe NPLs were separated from other reaction products with successive purification steps upon addition of hexane. First, the resulting mixture was centrifuged at $14500 \mathrm{rpm}$ for $10 \mathrm{~min}$, and the supernatant was removed from the centrifuge tube. The precipitate was dried under nitrogen and dissolved in hexane and centrifuged again at 4500 rpm for $5 \mathrm{~min}$. In the second step, the supernatant was separated into another centrifuge tube and ethanol was added into supernatant solution until it became turbid. In the last step, after the turbid solution was centrifuged at $4500 \mathrm{rpm}$ for $5 \mathrm{~min}$, the precipitate was dissolved in hexane and filtered with 0.20 $\mu \mathrm{m}$ filter.

Steady-State Photoluminescence Spectrum and Time-Resolved Fluorescence Measurements. Nonstacked NPL solution was spincoated onto a $1.2 \times 1.2 \mathrm{~cm}^{2}$ quartz substrate at $1000 \mathrm{rpm}$ for 30 s. Stacked NPL solution was drop-cast onto quartz with the same dimensions. The films were placed into a close-cycle $\mathrm{He}$ cryostat from Oxford Instruments. A $375 \mathrm{~nm}$ pulsed laser with a repetition rate of $2.5 \mathrm{MHz}$ was used to excite the samples. The excitation was coupled to the photodetector of the TCSPC device for TRF measurements and a fiber-optical cable connected to a Maya 2000 Pro spectrometer for the PL measurements at the same time. The lifetime decays have been numerically fit by multiexponential decays using FluoFit software in deconvolution mode.

\section{ASSOCIATED CONTENT}

\section{Supporting Information}

The Supporting Information is available free of charge on the ACS Publications website at DOI: 10.1021/acs.jpclett.5b02763.

Number of NPLs in stacks given as a histogram, calculation of the upper bound for RT QY of nonstacked NPLs using temperature-dependent PL spectra, and derivation of QY and average lifetime formulas used in simulations (PDF)

\section{AUTHOR INFORMATION}

\section{Corresponding Author}

*E-mail: volkan@bilkent.edu.tr, hvdemir@ntu.edu.sg. Phone: +90 312 290-1021. Fax: +90 312 290-1123.

\section{Notes}

The authors declare no competing financial interest.

\section{ACKNOWLEDGMENTS}

The authors acknowledge the financial support from Singapore National Research Foundation under the programs of NRF-RF2009-09 and NRF-CRP-6-2010-02 and the Science and Engineering Research Council, Agency for Science, Technology and Research (A*STAR) of Singapore; EU-FP7 Nano- 
photonics4Energy NoE; and TUBITAK EEEAG 109E002, 109E004, 110E010, 110E217, 112E183, and 114E410. H.V.D. acknowledges support from ESF-EURYI and TUBA-GEBIP.

\section{REFERENCES}

(1) Ithurria, S.; Tessier, M. D.; Mahler, B.; Lobo, R. P. S. M.; Dubertret, B.; Efros, A. L. Colloidal Nanoplatelets with TwoDimensional Electronic Structure. Nat. Mater. 2011, 10, 936-941.

(2) Tessier, M. D.; Biadala, L.; Bouet, C.; Ithurria, S.; Abecassis, B.; Dubertret, B. Phonon Line Emission Revealed by Self-Assembly of Colloidal Nanoplatelets. ACS Nano 2013, 7, 3332-3340.

(3) Kunneman, L. T.; Schins, J. M.; Pedetti, S.; Heuclin, H.; Grozema, F. C.; Houtepen, A. J.; Dubertret, B.; Siebbeles, L. D. A. Nature and Decay Pathways of Photoexcited States in CdSe and CdSe/CdS Nanoplatelets. Nano Lett. 2014, 14, 7039-7045.

(4) Yoffe, A. D. Low-Dimensional Systems: Quantum Size Effects and Electronic Properties of Semiconductor Microcrystallites (zeroDimensional Systems) and Some Quasi-Two-Dimensional Systems. Adv. Phys. 2002, 51, 799-890.

(5) Tessier, M. D.; Javaux, C.; Maksimovic, I.; Loriette, V.; Dubertret, B. Spectroscopy of Single CdSe Nanoplatelets. ACS Nano 2012, 6, 6751-6758.

(6) Guzelturk, B.; Erdem, O.; Olutas, M.; Kelestemur, Y.; Demir, H. V. Stacking in Colloidal Nanoplatelets: Tuning Excitonic Properties. ACS Nano 2014, 8, 12524-12533.

(7) Chen, Z.; Nadal, B.; Mahler, B.; Aubin, H.; Dubertret, B. Quasi2D Colloidal Semiconductor Nanoplatelets for Narrow Electroluminescence. Adv. Funct. Mater. 2014, 24, 295-302.

(8) Guzelturk, B.; Kelestemur, Y.; Olutas, M.; Delikanli, S.; Demir, H. V. Amplified Spontaneous Emission and Lasing in Colloidal Nanoplatelets. ACS Nano 2014, 8, 6599-6605.

(9) Grim, J. Q.; Christodoulou, S.; Di Stasio, F.; Krahne, R.; Cingolani, R.; Manna, L.; Moreels, I. Continuous-Wave Biexciton Lasing at Room Temperature Using Solution-Processed Quantum Wells. Nat. Nanotechnol. 2014, 9, 891-895.

(10) Olutas, M.; Guzelturk, B.; Kelestemur, Y.; Yeltik, A.; Delikanli, S.; Demir, H. V. Lateral Size-Dependent Spontaneous and Stimulated Emission Properties in Colloidal CdSe Nanoplatelets. ACS Nano 2015, 9, 5041-5050.

(11) Abécassis, B.; Tessier, M. D.; Davidson, P.; Dubertret, B. SelfAssembly of CdSe Nanoplatelets into Giant Micrometer-Scale Needles Emitting Polarized Light. Nano Lett. 2014, 14, 710-715.

(12) Jana, S.; Phan, T. N. T.; Bouet, C.; Tessier, M. D.; Davidson, P.; Dubertret, B.; Abécassis, B. Stacking and Colloidal Stability of CdSe Nanoplatelets. Langmuir 2015, 31, 10532-10539.

(13) Sillen, A.; Engelborghs, Y. The Correct Use of "Average" Fluorescence Parameters. Photochem. Photobiol. 1998, 67, 475-486.

(14) Guzelturk, B.; Martinez, P. L. H.; Zhang, Q.; Xiong, Q.; Sun, H.; Sun, X. W.; Govorov, A. O.; Demir, H. V. Excitonics of Semiconductor Quantum Dots and Wires for Lighting and Displays. Laser Photon. Rev. 2014, 8, 73-93.

(15) Guzelturk, B.; Olutas, M.; Delikanli, S.; Kelestemur, Y.; Erdem, O.; Demir, H. V. Nonradiative Energy Transfer in Colloidal CdSe Nanoplatelet Films. Nanoscale 2015, 7, 2545-2551.

(16) Achtstein, A. W.; Schliwa, A.; Prudnikau, A.; Hardzei, M.; Artemyev, M. V.; Thomsen, C.; Woggon, U. Electronic Structure and Exciton-Phonon Interaction in Two-Dimensional Colloidal CdSe Nanosheets. Nano Lett. 2012, 12, 3151-3157.

(17) Montroll, E. W. Random Walks on Lattices. III. Calculation of First-Passage Times with Application to Exciton Trapping on Photosynthetic Units. J. Math. Phys. 1969, 10, 753-765.

(18) Lutz, D. R.; Nelson, K. A.; Gochanour, C. R.; Fayer, M. D. Electronic Excited State Energy Transfer, Trapping by Dimers and Fluorescence Quenching in Concentrated Dye Solutions: Picosecond Transient Grating Experiments. Chem. Phys. 1981, 58, 325-334.

(19) Anikeeva, P. O.; Madigan, C. F.; Halpert, J. E.; Bawendi, M.; Bulović, V. Electronic and Excitonic Processes in Light-Emitting
Devices Based on Organic Materials and Colloidal Quantum Dots. Phys. Rev. B: Condens. Matter Mater. Phys. 2008, 78, 085434.

(20) Kułak, L.; Bojarski, C. Forward and Reverse Electronic Energy Transport and Trapping in Solution. I. Theory. Chem. Phys. 1995, 191, $43-66$.

(21) Kułak, L.; Bojarski, C. Forward and Reverse Electronic Energy Transport and Trapping in Solution. II. Numerical Results and Monte Carlo Simulations. Chem. Phys. 1995, 191, 67-86.

(22) Sanchez, A.; Dominguez-Adame, F.; Macia, E. Excitation Decay in 1D Disordered Systems. Phys. Rev. B: Condens. Matter Mater. Phys. 1995, 51, 173-178.

(23) She, C.; Fedin, I.; Dolzhnikov, D. S.; Demortière, A.; Schaller, R. D.; Pelton, M.; Talapin, D. V. Low-Threshold Stimulated Emission Using Colloidal Quantum Wells. Nano Lett. 2014, 14, 2772-2777.

(24) Förster, T. Zwischenmolekulare Energiewanderung Und Fluoreszenz. Ann. Phys. 1948, 437, 55-75.

(25) Rowland, C. E.; Fedin, I.; Zhang, H.; Gray, S. K.; Govorov, A. O.; Talapin, D. V.; Schaller, R. D. Picosecond Energy Transfer and Multiexciton Transfer Outpaces Auger Recombination in Binary CdSe Nanoplatelet Solids. Nat. Mater. 2015, 14, 484-489.

(26) Lorenzon, M.; Christodoulou, S.; Vaccaro, G.; Pedrini, J.; Meinardi, F.; Moreels, I.; Brovelli, S. Reversed Oxygen Sensing Using Colloidal Quantum Wells towards Highly Emissive Photoresponsive Varnishes. Nat. Commun. 2015, 6, 6434.

(27) Tessier, M. D.; Mahler, B.; Nadal, B.; Heuclin, H.; Pedetti, S.; Dubertret, B. Spectroscopy of Colloidal Semiconductor Core/Shell Nanoplatelets with High Quantum Yield. Nano Lett. 2013, 13, 33213328.

(28) Prudnikau, A.; Chuvilin, A.; Artemyev, M. CdSe-CdS Nanoheteroplatelets with Efficient Photoexcitation of Central CdSe Region through Epitaxially Grown CdS Wings. J. Am. Chem. Soc. 2013, $135,14476-14479$.

(29) Kelestemur, Y.; Olutas, M.; Delikanli, S.; Guzelturk, B.; Akgul, M. Z.; Demir, H. V. Type-II Colloidal Quantum Wells: CdSe/CdTe Core/Crown Heteronanoplatelets. J. Phys. Chem. C 2015, 119, 21772185.

(30) Tessier, M. D.; Spinicelli, P.; Dupont, D.; Patriarche, G.; Ithurria, S.; Dubertret, B. Efficient Exciton Concentrators Built from Colloidal Core/Crown CdSe/CdS Semiconductor Nanoplatelets. Nano Lett. 2014, 14, 207-213.

(31) Feldmann, J.; Peter, G.; Göbel, E. O.; Dawson, P.; Moore, K.; Foxon, C.; Elliott, R. J. Linewidth Dependence of Radiative Exciton Lifetimes in Quantum Wells. Phys. Rev. Lett. 1987, 59, 2337-2340. 